\title{
An overview of media streams caching in peer-to-peer systems
}

\begin{abstract}
Nowadays, the idea of media contents streaming through the Internet has become a very important issue. On the other hand, full caching for media objects is not a practical solution and leads to consume the cache storage in keeping few media objects because of its limited capacity. Furthermore, repeated traffic which is being sent to clients wastes the network bandwidth. Thus, utilizing the bandwidth of the network is considered as an important objective for network administrators. Media objects have some characteristics that have to be considered when a caching algorithm is going to be performed. In this paper, recent approaches that have been proposed for media streams caching in peer-to-peer systems are reviewed.
\end{abstract}

Keyword: Media streaming; Caching; Peer-to-peer systems; Proxy servers 